\section{Favorable Response to Mirtazapine in John Cunningham Virus-related Gray Matter Lesion in a Patient with Human Immunodeficiency Virus}

\author{
Abdullah I. Alwehaibi ${ }^{1}$, Mohammed I. AlJaber ${ }^{2}$, Shahpar Nahrir ${ }^{3}$ \\ 1. Surgery, King Saud Medical City, Riyadh, SAU 2. Miscellaneous, Al-Imam Mohammad Ibn Saud Islamic University, \\ Riyadh, SAU 3. Neurology, King Saud Medical City, Riyadh, SAU
}

Corresponding author: Shahpar Nahrir, snahrir14@gmail.com
Received 02/04/2019

Review began 02/25/2019 Review ended 03/10/2019 Published 03/14/2019

\section{() Copyright 2019}

Alwehaibi et al. This is an open access article distributed under the terms of the Creative Commons Attribution License CC-BY 3.0., which permits unrestricted use, distribution, and reproduction in any medium, provided the original author and source are credited.

\begin{abstract}
Mirtazapine has recently emerged as a promising agent for the treatment of progressive multifocal leukoencephalopathy (PML). While there is no Class I evidence for its use, numerous case reports have illustrated mirtazapine's efficacy. True to its name, PML is known to occur mostly in the white matter of the brain as its causative agent, John Cunningham virus (JC virus), has a predilection for infecting glial cells. The virus replicates vigorously in oligodendrocytes and causes lysis of the glial cell culminating in demyelination. Therefore, gray matter involvement is rare. Mirtazapine's 5HT2A receptor blocking capacity is presumed to hinder JC virus' entry into glial cells. We report a case of a patient with human immunodeficiency virus (HIV) with predominantly gray matter lesions from JC virus reactivation. This case is the first reported case of gray matter PML in an Arabic patient who responded favorably to mirtazapine therapy.
\end{abstract}

Categories: Neurology, HIV/AIDS, Infectious Disease

Keywords: jcv, pml, mirtazapine, gray matter, hiv/aids, favorable response, arab

\section{Introduction}

John Cunningham virus (JC virus) is notorious for being highly lethal. Non-archetype JC virus is harmful to brain cells. Archetypal JC virus is ubiquitous in distribution; its worldwide seroprevalence is approximately 80\% [1]. JC virus deoxyribonucleic acid (DNA) has been detected in oligodendrocytes, astrocytes, lymphocytes, kidney epithelium cells, tonsil stromal cells, and plasma cells of healthy individuals and is periodically shed in urine [2-3].

The virulence of JC virus is established when extensive deletions and duplications in the nucleotide sequences within the promoter/enhancer region of the genome occur [4]. The resultant pathogenic variant acquires a tandem repeat of a 98-bp element (various Mad strains) [5]. This process is triggered by a loss of protective cellular immunosurveillance. This pathogenic variant, upon entry into glial cells, causes extensive myelin destruction. As it commonly affects the oligodendrocytes, lesions are seen more frequently in the white matter. However, gray matter is also composed of (to a lesser extent) some glial cells and myelinated fibers [5]. Therefore, JC virus-related deep gray matter lesions are not impossible.

Nevertheless, wherever it affects in the brain, JC virus leads to disastrous consequences. In the era before highly active antiretroviral therapy, the prognosis for patients with progressive multifocal

leukoencephalopathy (PML) was grave; death occurred in approximately 95\% of patients within four to six months of diagnosis [6]. Those who survive PML can be left with severe neurological disabilities. Despite the dire prognosis, there is no specific established treatment modality for PML. There is, however, is an urge in the scientific community to explore different agents to counteract the deleterious effects of JC virus. One such endeavor is the use of the 5HT2A receptor blocker mirtazapine, and the successful use of mirtazapine has been reported in several case reports. We present a case where a male patient of Arab ancestry diagnosed with acquired immunodeficiency syndrome (AIDS) was found to have basal ganglion and thalamic lesions who responded remarkably to mirtazapine therapy.

\section{Case Presentation}

A 37-year-old Yemeni male patient was brought to the hospital by his coworker after the patient had been disoriented for several days. A report from another medical center indicated the patient was diagnosed with tuberculosis (TB). According to this report, he had presented to the other facility one month previous with fever, productive cough, and disorientation. He was advised to start anti-TB therapy (ATT). However, the lack of collateral history made it impossible to confirm whether he had been receiving ATT. At presentation to our center, he was drowsy but arousable to strong verbal commands. He uttered a few incomprehensible sounds, and he obeyed spoken commands only occasionally. He appeared to have mild weakness in his left arm. His cranial nerve examination showed no abnormalities. The results of his systemic examination were unremarkable: we noted no skin rash, indications of intravenous drug use, evidence of peripheral stigmata 


\section{Cureus}

of infective endocarditis, or lymphadenopathy. On subsequent days, he sustained several attacks of generalized tonic-clonic seizures. Given that he was febrile with evidence of meningismus, he underwent lumbar tap.

\section{Investigations}

His cerebrospinal fluid (CSF) was light yellowish with 373 cells (73\% monomorphic) with glucose at $2.4 \mathrm{~g} / \mathrm{dL}$, protein at $620 \mathrm{~g} / \mathrm{dL}$. The CSF was positive for JC virus DNA with a viral load of $2800 \mathrm{copies} / \mathrm{mL}$. His serology for human immunodeficiency virus (HIV) was positive with a CD4 count of $135 / \mu \mathrm{L}$ and an HIV viral load of 179,795 copies/mL. Test results for hepatitis B virus and sputum acid-fast bacilli were negative. CSF polymerase chain reaction results for TB were negative, so was the culture for TB in the CSF sample. However, the report from the peripheral center had shown positive TB culture in CSF. Computed tomography (CT) of the patient's brain revealed left basal ganglia hypodensity and left periventricular white matter hypodensity. Magnetic resonance imaging (MRI) showed bilaterally scattered patchy confluent subcortical and periventricular foci confirmed on T2-weighted images (T2WI) and fluid-attenuated inversion recovery (FLAIR) images (Figure 1). We noted diffusion-weighted imaging (DWI) restriction at the left frontal lobe, bilateral basal ganglia as well as in the brainstem and cerebellar vermis. The largest area of DWI restriction was seen over the right basal ganglia lesion which also showed faint heterogeneous enhancement in the post-contrast images with mild mass effect on the adjacent right lateral ventricle.

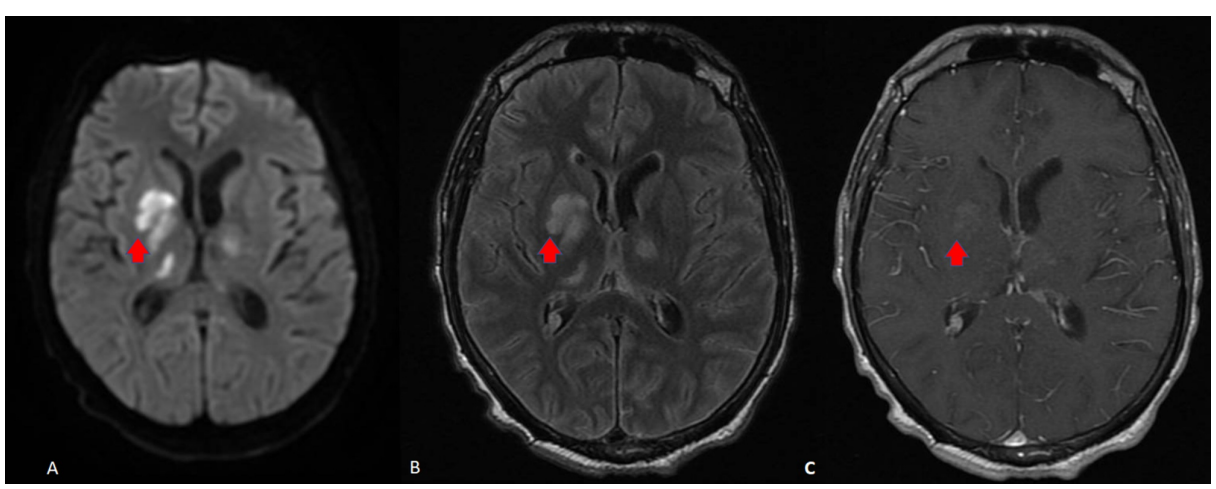

\section{FIGURE 1: Brain magnetic resonance imaging (MRI) at presentation, prior to treatment with mirtazapine}

A) diffusion-weighted imaging (DWI); B) fluid-attenuated inversion recovery (FLAIR); C) contrast study. Lesion visible over deep gray matter of basal ganglion and thalami.

\section{Treatment}

Based on clinical, imaging, and virological finding, we decided to treat the patient with combined antiretroviral therapy (cART) along with the ATT. Given the reported cases of successful treatment with an anti-5HT2A antagonist, we treated the patient with mirtazapine along with standard cART and ATT.

\section{Outcome}

The patient remained clinically stable with no apparent worsening of his neurological deficit. He became more alert and could reply to one or two questions coherently. He sustained no further seizures. He developed rigidity in all limbs and bradyphrenia. He also became hypophonic and apathetic. A second MRI after six weeks of therapy revealed remarkable improvement (Figure 2). After six months of treatment, the patient is more alert, obeys commands, and can communicate with two to three sentences at a time. His weakness has resolved, but he has residual rigidity over his limbs. 




FIGURE 2: Brain magnetic resonance imaging (MRI) six weeks after mirtazapine therapy

A) T2-weighted images (T2WI) showing marked resolution of previously seen lesion over gray matter; B) fluidattenuated inversion recovery (FLAIR) image demonstrating similar resolution of lesion size; C) contrast study shows no discernible area of contrast uptake.

\section{Discussion}

PML is a demyelinating disease caused by JC virus, a polyomavirus that affects CNS white matter. This disease was first noted by Astrom et al. in 1958 based on the characteristic histopathological features of demyelination, bizarre astrocytes, and enlarged oligodendroglia nuclei [7]. JC virus was later isolated from the brain of a patient named John Cunningham in 1971. JC virus vigorously replicates in glial cells and results in extensive myelin breakdown and white matter destruction. The virus is thought to act on the 5HT2A receptor on the glial cells to gain entry into the cell [8]. 5HT2 receptors are expressed in the brain microvasculature and on astrocytes at the blood-brain barrier [9]. In the pre-HIV era, PML was infrequent and occurred mostly in patients who were immunocompromised from underlying hematologic malignancies or immunosuppressive medications, or transplant recipients and patients of advanced age [7]. In the last four decades, PML has occurred in greater frequency in AIDS patients compared with patients in an immunosuppressed state from other conditions [10]. The first description of PML in an AIDS patient was reported in 1982 [11]. The estimated risk of developing PML in AIDS patients range from 1\% to 4\% [12]. A study in the U.S. showed that $82 \%$ of the 9,675 PML cases documented from 1998 to 2005 were associated with HIV infections [13]. PML is known to occur in both HIV-1 and HIV-2 infected patients. Akin to our patient, most HIV-infected patients develop PML as they approach a poor immunological status expressed by a low CD4 cell count (<200/ $\mu \mathrm{L})$ [14-15]. The fundamental pathological feature of PML is demyelination [16]. Demyelination typically occurs as a multifocal process; it can occur in any location in the white matter and range in size from $1 \mathrm{~mm}$ to several centimeters [16]. A JC virus infection in gray matter structures such as the basal ganglia or thalamus is a rare occurrence. Theoretically, a JC virus infection in gray matter is plausible as gray matter also possess some degree of myelinated fiber and glial cells. Lesions of the deep gray structures were noted in a study of $47 \mathrm{HIV}$ patients with PML, and basal ganglia lesions were found in $7 \%$ of the patient and thalamic lesions were found in $8 \%$ [17]. The common clinical findings are behavioral and cognitive abnormalities which are seen in one-third to one-half of all patients. Motor weakness, gait abnormalities, visual field deficits, speech and language disturbances, and incoordination are also seen in PML [16]. Sensory loss, seizures, headache, and diplopia occur less frequently [16]. In neuroimaging, MRI shows hyperintense lesions on T2WI and FLAIR images in the affected regions [16]. Classically, PML lesions do not show edema, mass effect or contrast enhancement on imaging [5]. The resultant infection generally remains refractory to treatment.

There is currently no specific treatment available for this catastrophic illness. However, the pragmatic approach targets the basic mechanism of action of the virus. This includes immune response modulator agents that inhibit JC virus entry into the glial cells and inhibit JC virus DNA replication [18]. Combined antiretroviral therapy is considered the first-line of therapy as they are believed to counteract JC virus DNA replication and aid in the restoration of the adaptive immune response to JC virus. However, a plethora of other agents with different indications have surfaced recently as promising JC virus glial cell entry inhibitors. Mirtazapine is one of these reported drugs with demonstrated success in a small series of HIVpositive PML cases [19-20]. Mirtazapine is an alpha-2-adrenergic, 5HT2A- and 5HT3-receptor antagonist that can cross the blood-brain barrier [19]. By limiting JC virus entry into the glial cells, mirtazapine may retard the adverse effect of its infection. While the mechanism is promising, a study published in 2016 systemically reviewed data from 2005 to 2015 of five cohort studies and 74 case reports found only the natalizumab-induced PML cases responded positively to mirtazapine treatment [19]. 
Our case presents a favorable response to mirtazapine in an HIV-related, predominantly gray matter affected PML. Nevertheless, as this case had quite a few atypical features without a histopathological confirmation of PML therefore, we can not completely rule out having other less likely differentials for the lesions seen in MRI brain and for the rapid response to combination treatment (cART, Mirtazapine, and ATT). In other words, it may not be incorrect to presume that there was concomitant CNS TB and restoration of ATT use may have influenced the quick resolution of the brain lesions. However, as CSF TB culture was negative at our center, we were hesitant to make the diagnosis of TB. Instead, in our patient, JC virus DNA was detected by PCR in the CSF, the presence of which is generally considered highly specific for the diagnosis of PML. Therefore, we feel it was most likely a case of predominantly gray matter involved PML in an HIV positive patient.

\section{Conclusions}

This case represents the first report of PML in the gray matter of an Arab patient with HIV who responded well to mirtazapine treatment in conjuction with cART.

\section{Additional Information \\ Disclosures}

Human subjects: Consent was obtained by all participants in this study. Conflicts of interest: In compliance with the ICMJE uniform disclosure form, all authors declare the following: Payment/services info: All authors have declared that no financial support was received from any organization for the submitted work. Financial relationships: All authors have declared that they have no financial relationships at present or within the previous three years with any organizations that might have an interest in the submitted work. Other relationships: All authors have declared that there are no other relationships or activities that could appear to have influenced the submitted work.

\section{References}

1. Bellizzi A, Anzivino E, Rodio DM, Palamara AT, Nencioni L, Pietropaolo V: New insights on human polyomavirus JC and pathogenesis of progressive multifocal leukoencephalopathy. Clin Dev Immunol. 2013, 1-17. 10.1155/2013/839719

2. Chapagain ML, Nerurkar VR: Human polyomavirus JC (JCV) infection of human B lymphocytes: a possible mechanism for JCV transmigration across the blood-brain barrier. J Infect Dis. 2010, 202:184-91. $10.1086 / 653823$

3. Kitamura T, Sugimoto C, Kato A, et al.: Persistent JC virus (JCV) infection is demonstrated by continuous shedding of the same JCV strains. J Clin Microbiol. 1997, 35:1255-7.

4. Johnson EM, Wortman MJ, Dagdanova AV, Lundberg PS, Daniel DC: Polyomavirus JC in the context of immunosuppression: a series of adaptive, DNA replication-driven recombination events in the development of progressive multifocal leukoencephalopathy. Clin Dev Immunol. 2013, 2013:197807. 10.1155/2013/197807

5. Tan CS, Koralnik IJ: Progressive multifocal leukoencephalopathy and other disorders caused by JC virus: clinical features and pathogenesis. Lancet Neurol. 2010, 9:425-37. 10.1016/S1474-4422(10)70040-5

6. Berger JR, Major EO: Progressive multifocal leukoencephalopathy. Semin Neurol. 1999, 19:193-200. 10.1055/s-2008-1040837

7. Astrom KE, Mancall EL, Richardson Jr EP, Zu Rhein G: Progressive multifocal leukoencephalopathy (PML): a 50-years old disease. Brain. 1958, 81:93-111.

8. Elphick GF, Querbes W, Jordan JA, et al.: The human polyomavirus JCV uses serotonin receptors to infect cells. Science. 2004, 306:1380-3. 10.1126/science.1103492

9. Susama V, Katia C, Koralnik IJ, et al.: Mirtazapine in progressive multifocal leukoencephalopathy associated with polycythemia vera. J Infect Dis. 2007, 196:709-11. 10.1086/520514

10. Sacktor N, Lyles RH, Skolansky R, et al.: HIV-associated neurologic disease incidence changes: multicenter AIDS cohort study, 1990-1998. Neurology. 2001, 56:257-260. 10.1212/WNL.56.2.257

11. Miller JR, Barrett RE, Britton CB, et al.: Progressive multi-focal leukoencephalopathy in a male homosexual with T-cell immune deficiency. New Engl J Med. 1982, 307:1436-8. 10.1056/NEJM198212023072307

12. Brooks BR, Walker DL: Progressive multifocal leukoencephalopathy. Neurol Clin. 1984, 2:299-313.

13. Molloy ES, Calabrese LH: Progressive multifocal leukoencephalopathy: a national estimate of frequency in systemic lupus erythematosus and other rheumatic diseases. Arthritis Rheum. 2009, 12:3761-5. 10.1002/art.24966

14. Balushi AA, Thomas FP: Progressive multifocal leukoencephalopathy in HIV. J Med. 2016, Accessed: January 23, 2019: https://emedicine.medscape.com/article/1167145-overview.

15. Mascarello M, Lanzafame M, Lattuada E, Concia E: Ferrari S: Progressive multifocal leukoencephalopathy in an HIV patient receiving successful long-term HAART. J Neurovirol. 2011, 2:196-9. 10.1007/s13365-0110020-y

16. Berger JR, Aksamit AJ, Clifford DB, et al.: PML diagnostic criteria: consensus statement from the AAN Neuroinfectious Disease Section. Neurology. 2013, 80:1430-8. 10.1212/WNL.0b013e31828c2fa1

17. Whiteman ML, Post MJ, Berger JR, Tate LG, Bell MD, Limonte LP: Progressive multifocal leukoencephalopathy in 47 HIV-seropositive patients: neuroimaging with clinical and pathologic correlation. Radiology. 1993, 187:233-240. 10.1148/radiology.187.1.8451420

18. Pavlovic D, Patera AC, Nyberg F, Gerber M, Liu M: Progressive multifocal leukoencephalopathy: current treatment options and future perspectives. Ther Adv Neurol Disord. 2015, 8:255-73.

$10.1177 / 1756285615602832$ 


\section{Cureus}

19. Cettomai D, McArthur JC: Mirtazapine use in human immunodeficiency virus-infected patients with progressive multifocal leukoencephalopathy. Arch Neurol. 2009, 66:255-258. 10.1001/archneurol.2008.557

20. Mullins C, Miranda J, Sandoval H, Ramos-Duran L, Tonarelli SB: The benefit of mirtazapine in the treatment of progressive multifocal leukoencephalopathy in a young HIV-positive patient: a case report. Innov Clin Neurosci. 2018, 15:33-35. 